\title{
The Context and Long-term Impacts of Multiple Birth Loss: A Peer Support Network Perspective
}

\author{
Jean Kollantai \\ Center for Loss in Multiple Birth (CLIMB), Inc
}

Eollowing my own experience with the death of a twin baby, - I founded the Center for Loss in Multiple Birth (CLIMB) Inc. During the last 13 years I have worked with nearly 7000 bereaved parents of twins, triplets or other high multiples throughout the US and worldwide. The role of a peer support organisation and the range of families with multiples who have contacted it is described. They come from diverse backgrounds with many forms of bereavement: death during pregnancy, at birth or after of both or all babies, of one twin, of one or more higher order multiples; multifetal pregnancy reduction or selective fetocide; the death of one or more multiples in childhood. Some have had more than one multiple pregnancy and loss. Many are concerned for the surviving children. A peer support organisation has an important role to play in increasing public awareness of the needs of these families as well as influencing policy on infertility treatments that carry a high risk of producing multiple pregnancies with the associated risk of death and disability for one or more of the babies.

\section{The Formation of an Organization}

While society pays increasing attention to the joys and challenges of multiple births, a growing number of families are experiencing a dark and problematic side of the world of multiples. It is the death of one or more, both or all, of their babies during pregnancy, at birth, in infancy or in childhood. This was my own family's experience in 1986. Following the delivery of full term, fraternal twin boys after an uneventful pregnancy, I found myself holding one baby in each arm — one alive, and one equally handsome and big, but dead.

Shortly afterwards, I began to search for what I imagined would be only one or two others who had experienced this bittersweet, often agonizing, kind of parenthood. The next year I met Lisa Fleischer. Her twin son Teddy was an 8$1 / 2$ pound baby who died in the NICU following a prolapsed cord. Several months later, we met Rebecca Crandall whose identical baby girls died at 37 weeks gestation, for no known reason. Between the three of us we had expected six healthy, full term twin babies yet only two of them came home.

Following these meetings and with the support of the Anchorage Parents of Twins and Multiples Club, we founded the Center for Loss in Multiple Birth (CLIMB) Inc. In the 13 years since, we have been in contact with nearly 7000 bereaved multiple birth parents throughout the United States, Canada, Australia and New Zealand. CLIMB has developed and run a contact network, produced a newsletter and other resources for parents, caregivers and multiples groups, as well as organizing some local and regional gatherings. We hear from at least five more parents a day who are looking for information and support concerning losses which occurred any time up to 20 years ago. Some remain in touch for ten years or more. We also have many approaches from concerned relatives, friends and caregivers as well as the media and general public.

CLIMB families come from diverse socioeconomic and personal backgrounds, from 17-year-old single mothers living at home to someone of 52 expecting twins after losing one set last year. We have members living on state assistance or unemployed, and we have doctors, nurses, bankers and attorneys. There are a number of psychotherapists and social workers all of whom say that their professional background could never have prepared them for what they are now going through. Many have no living children, and some have no chance of ever having one, whereas others have as many as nine living children. There are a number of samesex couples who have used fertility technology to have children. There is a wide variety of racial and ethnic backgrounds, religious and other world views.

\section{Subgroups of Multiple Birth Loss}

There is also diversity in the kinds of multiple birth loss. Very many outcomes and combinations of events are possible, especially with the increasing use and poor regulation of fertility treatments. Several main subgroups can be identified within our group and contacts:

\section{The Death of All Multiples}

Over one-third of the parents have experienced the death of both their twins, or all of their triplets or higher multiples. Nothing is quite as heart wrenching as a call from someone having to bury all four or five babies after years of infertility, and then have to come home to what many call the

Address for correspondence: Jean Kollantai, Center for Loss in Multiple Birth (CLIMB), Inc. P.O. Box 91377 Anchorage AK 99509, USA.Email: climb@pobox.alaska.net 
"deafening silence" (Crandall, 1988). Many of the contacts in this situation are actually from fathers, as the mother is too distraught to reach out.

Many of these complete losses are due to prematurity or in-utero death and it is important for caregivers to remember that 22 weeks or even 13 weeks, feels a long way into the pregnancy for those who have gone through infertility for years and watched their babies' development through sonograms since six weeks. Prior to their loss, parents may have had complete baby showers in preparation for a large new family, having been told little or nothing of the risks and uncertainties.

Some bereavements follow multifetal pregnancy reduction from three or more fetuses. Twin to Twin Transfusion Syndrome (TTTS), even when treated, is a major cause of mortality, and other conditions unique to identicals such as conjoined and monoamniotic twins, are an enormous source of tragedy. We have also encountered hundreds of families who have lost both of their full term twins to sudden death in-utero, almost all of them monozygotic twins. Still other parents have lost both after a seemingly uneventful birth. Several CLIMB families have lost both twin babies to SIDS, or to an accident.

Another complication in multiple birth is that one family may experience (sometimes from unrelated causes) first an in-utero death, then a death at birth and then a death in the neonatal period, all in the context of one pregnancy. Facing the death of a baby one week, another the next week, and then next month the loss of the one child that you still had hopes for, is something that few parents would experience in a lifetime of tragedies.

Often, because of struggles with infertility, couples feel compelled to try again as soon as possible, not daring to wait a while. For those fortunate enough to conceive again, or to adopt, there is often a situation several years later which mirrors that of those who lose one of twins - the significant grieving for the baby who died earlier coupled with the current task of raising young children. These living children may even have resulted from the same batch of embryos as the original multiples, having been frozen for several years.

\section{The Death of One Twin}

These families represent over half of our group and Patricia Swanson has addressed many of the issues that face these parents. They may have experienced the death of a twin during pregnancy, in the neonatal period, or in the first year due to SIDS, or later with an acute or chronic illness or following an accident.

With ultrasound, many parents who would not previously have known they had lost a baby, are aware of the so-called vanishing twin syndrome - and may be heartbroken. Many had not been warned of the high risk of such a loss. More families with premature twins are now seeing their babies survive birth but then dying months or even a year or more later because technology has prolonged their life but was not ultimately successful. Many families have lost a twin or triplet toddler or child through drowning and household accidents.
We are also in contact with families who have a distressing ongoing twin pregnancy. One of their babies may already have died in-utero or they may already know that one baby has a lethal defect and is not likely to survive. We also receive many contacts from people trying to decide whether to have a multifetal pregnancy reduction; or whether to choose selective fetocide because one of their twins has a lethal defect or other serious complications which may compromise the other baby. This dilemma is unique to multiples because the lives and welfare of other, equally wanted, children are directly involved.

\section{Loss in Higher Multiple Births}

This, third major category of loss that we see is discussed by Elizabeth Bryan (p. 169).

\section{Repeated Multiple Pregnancies and Losses}

An increasing number of couples with repeated multiple pregnancies and multiple birth losses are now contacting us. Some parents have had as many as four twin pregnancies with a variety of outcomes, and others up to 15 conceptions in their childbearing years and have but a tiny, developmentally delayed survivor. A number of members have had twins or triplets subsequent to losing one or more baby from their first set of multiples. Conversely, many families have had their first pair of twins successfully but then lost one or both of a subsequent set.

Such combinations of experiences - all high risk and often medically traumatic to the mother as well - are made possible by fertility technology, but are outside any couple's usual expectations of pregnancy, childbirth and parenting. We have members who have lost both a set of twins and of triplets, as well as those who have lost both twins in one pregnancy, followed by one twin in the next.

\section{Emotional Impacts of Multiple Birth Loss}

Having also worked extensively for over 12 years in Alaska with peer support after singleton pregnancy and infant loss, I see two notable differences about the loss in a multiple birth. First there is the complexity and the timing of the grief process, and then a kind of pervasive confusion. The complexity is inherent in the number of babies at issue, and the fact that there may have been complicated courses of events for each baby, for the pregnancy and birth as a whole, and for the mother medically. Because of the realities of caring for a tiny survivor or survivors (or the preoccupation of another pregnancy soon after the death of all their children) parents are typically unable to grieve properly while caring for other babies and young children. They often cannot truly begin the grieving process until the survivors or subsequent children and the situation in general is somewhat stabilized.

It is not uncommon, therefore, for mothers to contact us whose baby or babies died several years earlier. They express feelings of depression, anxiety, and of not being sufficiently available to their living children. They are also often at a loss about how to talk to them about their deceased siblings.

One such mother with two surviving triplets and two other children — all under the age of three — said her 
husband was afraid that if she grieved openly about the triplet who died, she would become "obsessed" and unable to care for the living children. She, herself, expressed fears that it was exactly if she did not have the opportunity to mourn for her son, that she would become obsessed and unable to function.

When parents do stop having children they need to process their childbearing experience as a whole and deal with any unfinished business. Many face unanticipated parenting issues now that they have several living children. Those who have multiples a second time find themselves feeling much like those with a surviving twin — grateful for their children but seeing even more clearly what they have missed and finding that no one person, or set of persons, can truly replace another.

Normal grief after a multiple birth loss is often more difficult not only due to the pressures to be "ok" - especially with the surviving children - but again, because of the complexity of these situations. There is for example a greater likelihood that the parents never held both or all of their babies or held them together. Sometimes they did not even see them. One or both parents may not have been able to be present when one or more was baptized or died. Fathers and mothers of multiples are often separated, having to divide their attention between different babies. When a baby has died in utero, there are difficult questions about whether even to see the baby or babies, and then later, about whether they truly existed. It is very difficult to say goodbye to one's babies without first having said hello.

Caregivers and others who are well used to singleton loss may become confused or overwhelmed by multiple birth loss. Care by people who acknowledge and appreciate the complexity and scope of the loss - even when parents have had time and opportunity to have concrete experiences with all of their children - is by far the best foundation for helping parents to process their losses, to cope and heal in their own individual and holistic way over time.

CLIMB has developed special resource materials to help caregivers anticipate the needs of bereaved multiple birth parents in hospital settings (Kollantai \& Fleischer, 1993).

Parents' clinical experiences and the information given inevitably affects their later grief experiences and their ability to cope with them. Various obstacles to healthy healing need to be addressed; for example, the sense of isolation if they are made to feel that their type of loss is extremely rare. Their grief is often complicated by their lack of medical information on multiples imparted before treatment for infertility, at the time of diagnosis and through the pregnancy. This lack of information may be combined with the very uneven standard of care of multiple pregnancies and births. Most parents will have assumed there would be an established protocol for the management of a multiple pregnancy. Many find this is not so particularly in the less commons situations such as monoamniotic pregnancies.

\section{Issues Surrounding Surviving Children, and Families}

There are many issues in caring for a surviving multiple birth child. While there may well be some inherent effects on survivors of pregnancy and birth loss, surviving multiples usually seem to have normal psychological outcomes if their parents are able to deal with their own sense of loss in a way that enables them also to relate to their child's feelings and needs, rather than projecting their unexpressed grief onto their child (Pector, 2001). One extreme parental reaction to a multiple loss is that of never talking about the twin/multiple who died.

Many adult survivors of such situations have told us that they would rather their parents had said anything. They felt that their mother was not bonded to them and that it was therefore no wonder they were lonely and that they tried to imagine their twin was with them when their mothers were always looking at them and trying to "see" both children. They felt they were never enough.

Other parents so thoroughly envelope the survivor in idealized fantasies about his deceased twin and how wonderful it would have been, particularly at times such as birthdays and childhood firsts, that the whole family has serious problems. Many survivors, especially monozygotic twins, are taught to believe that their twin is living an exactly parallel existence to them up in the sky, looking down on them at all times but never reachable. There can be pressure on parents to see something tangible in the surviving child that demonstrates that $s /$ he is aware of his/her twinship; for example, parents often relate extreme shyness in survivors to the loss of a multiple sibling. Especially with first-time parents, it can take a great deal of support to enable them to avoid some of the pitfalls and to relate to a surviving multiple or subsequent children as individuals with their own temperament and needs. Those who have frail or handicapped survivors - which is quite common - have many additional challenges, including coping with a constant reminder of all the medical and emotional traumas experienced by the family.

CLIMB sees many mothers and fathers who are struggling with depression, anxiety, isolation, substance abuse, marital conflict and/or parenting problems which were at the very least exacerbated by their loss. In our experience, the first five years post-loss can be particularly stressful and there may still be significant difficulties over the following five years. Many need a great deal of time and support, as well as sensitive professional counseling. The impacts of a child's death last forever and are usually profound and life-changing.

Finally, the loss is accentuated by the preciousness and irreplaceability of twins and multiples. No parent can plan to have a multiple pregnancy again and even if they do, it tends to be terrifying. A subsequent single baby does not attract the same special attention and wonder that multiples inevitably bring. The experience of expecting or having twins or more is extraordinarily powerful and most people become totally enmeshed in the unique world of multiples. For some, they are seen as the "prize" of parenthood. Many who lose them therefore feel that they were first chosen only then to be cheated of an incredible oncein-a-lifetime opportunity. Encounters with intact sets of living multiples, especially those of the same number, sex and zygosity, are likely to be quite painful, even many years later. The yearning is exacerbated by the extreme 
media attention given to "successful" multiple pregnancies, especially sets of five or more.

\section{Conclusion}

A recent analysis of 10 years' birth data from Washington State showed that 1 of 20 women pregnant with twins at 20 weeks had lost one or both babies before the end of the perinatal period (in addition to loss before and after those periods) (Hartley et al., 2001). This means that parents may be far more likely to lose a baby than they were to conceive twins in the first place. CLIMB is disturbed that the numbers of multiple conceptions are rising faster than known risks can possibly decline. Considering the myriad long term implications of multiple birth losses as we see them every day, a medical imperative is needed to prevent these human tragedies and to mitigate the impacts when they do occur, including well informed and compassionate care for those parents who do experience death in multiple birth.

\section{Acknowledgements}

My special thanks to my husband and sons. My gratitude also goes to Elizabeth Bryan, MD, and others such as Elizabeth Noble, Patricia Malmstrom and Sr. Jane Marie Lamb of National SHARE for their help and encouragement in the founding of Our Newsletter and the formation of CLIMB. Edited for publication by Lisa Fleischer.

\section{References}

Crandall, R. (1988). Emily \& Julia. Our Newsletter, II(3), 12-13.

Hartley, R. S., Emanuel, I., \& Hitti, J. (2001). Perinatal mortality and neonatal morbidity rates among twin pairs at different gestational ages: Optimal delivery timing at 37 to 38 weeks' gestation. American Journal of Obstetrics and Gynecology, 184(3), 451-458

Kollantai, J., \& Fleischer, L. (1993). Multiple birth loss and the hospital caregiver. Palmer, Alaska: CLIMB, Inc.

Pector, E. (2001). Raising survivors of multiple birth loss: What can parents expect? Self-published (P.O. Box 5845, Naperville IL 60567). 